\title{
Mast cells and their role in inflammatory and tumor tissues
}

\begin{abstract}
Mast cells are multifunctional cells and they can also have a role as effector factors of the human innate and adaptive immune system. While a number of researchers still assume Mast cells to be augmenters of tumor growth, particularly via their effects on neoplastic angiogenesis; growing clinical and laboratory information also signify that Mast Cells could hamper tumor development. This inhibitory or promoting effect of Mast cells on tumor expansion seems to be depending on numerous relations among Mast cell, tumor cell type and the tumor stroma. Further in vitro and in vivo researches are required to elucidate more the interactions among Mast cells, tumor tissue and other inflammatory cells.
\end{abstract}

Keywords: Mast cell, Tumor, Cancer, Cytotoxicity, Neoangiogenesis, Inflammation
Volume 5 Issue 6 - 2017

\section{Oner Ozdemir}

Department of Pediatrics, Research and Training Hospital of Sakarya University, Turkey

Correspondence: Öner Özdemir, Division of Allergy and Immunology, Department of Pediatrics, Research and Training Hospital of Sakarya University, Faculty of Medicine, Sakarya University, Adnan Menderes Cad, Sagl?k Sok No: 195,Adapazari, Sakarya, Turkey, Tel 90-264-444-54-00, Fax 90-264-275-91-92, Email ozdemir_oner@hotmail.com

Received: August 22, 2017 | Published: August 28, 2017

\section{Introduction}

Findings of current researches imply a critical role for Mast cell (MC) in mediating host anti-tumor immune surveillance systemically and locally in the tumor stroma. And, the anti-tumor effect of human MC .MC-mediated cytotoxicity:MCC against various sensitive/resistant human leukemia/lymphoma cells was also shown by us in vitro supporting the emerging data in the literature. ${ }^{1,2}$ My interest in this review is that $\mathrm{MC}$ and its importance in tumor growth, neoplastic/tumor-associated angiogenesis as well as in tumor prognosis will be explained with a different perspective. ${ }^{1-9}$

\section{What is mast cell?}

Mast cell, a blood and connective tissue cell of the human and other vertebrates' immune system. Mast cells can intercede inflammatory (e.g. hypersensitivity and allergic) reactions in the human body. Mast cells are spread all through the various tissues of the body, particularly underneath the skin surface, around blood and lymphatic vessels, inside nerves, all the way through the airway system, and in the gastrointestinal and urinary systems. ${ }^{10}$ Mast cells accumulate and secrete many special mediators -such as histamine, interleukins, proteoglycans (e.g., heparin), and serine esterase enzymes (tryptase, chymase, granzymes, etc.) -in their granules of MC cytoplasm. After activation by an allergenic trigger, the $\mathrm{MC}$ releases the ingredients of their granules (also known as degranulation) into the adjacent tissues. These chemical mediators cause local and systemic responses, typical of an allergic / hypersensitivity reaction, e.g. augmented permeability of blood vessels (i.e., vasodilatation and anaphylactic shock), tightening of smooth muscles (e.g., bronchospasm), and amplified mucus secretion. ${ }^{11}$ Mast cells implicated in inflammatory and allergic (hypersensitivity) reactions have been understood until the midtwentieth century. Nevertheless, for the last several decades Mast Cells have been demonstrated to contribute to other immune system functions, together with innate and adaptive immune reactions. ${ }^{12}$

\section{What is the role of mast cells in inflamed and tumor tissue?}

Mast cell availability in various inflamed tissues including benign as well as malign lesions (tumors) and even in normal tissues raises the question about the actual role of MC. Mast Cells were also demonstrated to be augmented physiologically in the region of healing tissue, scars and ovulation. ${ }^{13}$ Since increased MCD is also seen with benign lesions such as hemangiomas; Mast Cells, in reality, just might be a manifestation of widespread inflammatory response of immune system in these areas. ${ }^{14-16}$ Also, the availability of Mast Cells in the tumor stroma could be just due to generalized inflammatory reaction indicating complex tumor-host relations favoring growth inhibitions depend on tumor type e.g. colorectal carcinomas. ${ }^{17}$ Some authors similarly think that the MCs may symbolize reactive cell types implicated in the pathophysiology of the host reaction, for instance, in early gastric cancer. ${ }^{18}$ In the tumor-induced inflammatory microenvironment, some of these proinflammatory mediators stimulate MC migration. MCs also mount up at sites of tumor progression in return to abundant chemoattractants e.g. CCL5/CXCR3 chemokines released by non-small cell lung carcinoma, and tumor-derived stem cell factor and CD30 expressions initiate MCD in lymphoma. ${ }^{15,19}$ As a result, only demonstrating high MCD in a tumor stroma having bad / good prognosis on biopsy sampling looks like unsatisfactory to clarify the genuine function of MC in those tissues. Then question is to be:are the MCs really mainly having an active function or are they just naive passerby secondarily in these tissues?

\section{Mast Cells in tumor tissue}

Albeit current literature evidence mostly suggests a contributing role for MCs in the growth of malignancies, rising data also implies that MCs may, indeed, have hampering roles against tumor cell growth relying on the tumor tissue microenvironment. Consistently, increased MCD was not persistently related with tumor progression/ bad prognosis/increased metastasis in established tumors such as in hepatocellular, colorectal and breast cancers. ${ }^{20-22}$ Previous researches have shown that mice with MC deficiency had an amplified tumor development when compared with control. This high tumor occurrence was decreased to the normal intensity once MC deficiency had been defeated. Additionally, tumor occurrence and progression were inversely correlated with $\mathrm{MC}$ density $(\mathrm{MCD})$ in the organs of MC-depleted, partially MC-depleted, and MC-sufficient mice. ${ }^{23,24} \mathrm{In}$ a study assessing increase in pulmonary metastases of B16 melanoma; lung metastases were considerably diminished in controls when compared to MC-free (W/Wv) mice. ${ }^{25}$ In another research performed 
with B16 melanoma cells of two strains of MC-deficient mice implied that the reliance of anti-tumor resistance on MCs. ${ }^{26}$

Mast Cells concentration in different tumor tissues is still controversial. And MCD has not always been reported as increased in tumor stroma of human as well as mice. In addition to noticeably reduced MCD during tumor progression in some cancers, there are other types in which MCD does not change. ${ }^{20,21}$ For instance:human hepatocellular, and some breast cancers as well as melanoma, lymphoplasmocytic lymphoma and chronic lymphoid leukemia. ${ }^{20-22}$ Moreover, there are some inconsistent literature revealing increased or decreased MCD in the same type of tumors such as NSCLC and colorectal carcinomas. ${ }^{27,28}$ Although it is difficult to clarify these contradictory results, they can be attributable to discrepancies in biopsy timing, type of tumor and methodology utilized for the detection of MCs in tumor tissues.

\section{Mast cell's cytotoxicity against tumor} cells:the antagonistic and dual role of mast cell's mediators in tumor growth as well as in neoangiogenesis

Mediators are shown to affect various features of tumor biology and the net consequence of $\mathrm{MC}$ in the tumor growth is hard to assess. Even though some MC mediators are demonstrated to antagonize tumor development and some others help to promote tumor growth. Below, this controversial issue is explained a little further.

The Importance of mast cell's mediators in tumor growth:the antagonistic role of mast cell's mediators in tumor progression

Higher MCD in various tumor tissues has been supposed to be related with more indolent tumor progression, signifying the likelihood of tumor inhibitory effects of MCs. Similarly, in recent literature data, $\mathrm{MC}$ gathering was suggested to restrain the growth and dissemination of the different carcinomas. ${ }^{6-8,20} \mathrm{MCs}$ are multifunctional cells and they are also known to act as effector components of the human immune system. Even though some authors still hypothesize MCs to be augmenters of tumor progression, in particular via their effects on neoangiogenesis; growing clinical and laboratory data also points to that MCs could restrain tumor expansion. First in vivo observations in the 1950's have proposed their potential function as anti-tumor effect in the localization of certain solid tumor types..$^{29}$ In vitro murine MCC against murine tumor cells was demonstrated in 1981 and it has been thought that murine MC has 'natural cytotoxicity' against susceptible murine cell lines (WEHI-164 and L929, etc.) by diverse death mechanisms, either reliant on mediator TNF- $\alpha$ or not such as proteases, leukotrienes and NO. ${ }^{30,31}$ Concomitantly; supporters of the MC's inhibitory role presume them as inhibitors of tumor growth by means of their pro-necrolytic/-apoptotic cytotoxic granules e.g. $\mathrm{TNF}-\alpha$ and serine proteases e.g. granzymes. Like other cytotoxickiller cells, MCs were also shown to have lavishly serine proteases termed granzymes counting several chymases in their granules, which is involved in cell-mediated cytotoxicity. Except for perforin, MCs indeed have been confirmed to include all components of cellmediated cytotoxicity. ${ }^{1,2}$ Our recent in vitro experiences showed human MCC against NK / LAK-sensitive / resistant human leukemia/ lymphoma cells. ${ }^{1,2}$

\section{The dual role of Mast cell's mediators in tumor growth}

There have been conflicting discussions in the literature on the function of MCs' mediators in tumor development. Some MC mediators such as chymase and tryptase may have also dual roles and the coexistence of these mediators yields to the tissue homeostasis. Although chymase is assumed to be an effective angiogenic and bad prognostic element; it was also been demonstrated to be related with cytotoxic effect against various type of cells e.g. in neonatal/adult animal cardiomyocytes, human vascular smooth muscle, and human conjunctival epithelial cells. ${ }^{32}$ Similarly; tryptase was considered to be a mediator resulting in fibrosis, thus preventing tumor expansion, besides an angiogenic factor, possibly helping tumor progression. ${ }^{20}$ As a result, MC mediators are able to provoke as well as hinder angiogenesis. ${ }^{33}$ Even like TNF- $\alpha$, some mediators such as tryptase, chymase and cathepsin $\mathrm{G}$ are thought to be accountable from both actions. ${ }^{34}$

\section{The importance of mast cell's mediators in neoangiogenesis}

\section{The antagonistic role of mast cell's mediators on tumor associated angiogenesis (neoplastic angiogenesis/ neoangiogenesis)}

Neoplastic angiogenesis (neoangiogenesis) seems to be a more intricate development than what has been defined, and the mechanism plus its significance are not elucidated. There have been lots of clinicohistopathological samples reported; however conflicting clinic and laboratory data on MC's role has been increasing in the literature. In contrary to hypothesis of MC-induced angiogenesis via mediators, MC-mediated apoptosis of vascular endothelial cells was also reported in vitro. ${ }^{35}$ In some mice as well as in human cancer studies, there was no considerable link between MCD and angiogenesis, for instance, lung cancer. ${ }^{14,27,28}$ According to a different aspect, tumorinduced inflammatory response counting macrophages and fibroblasts can create a local microenvironment causing tumor expansion via angiogenesis; and some of these angiogenic elements may motivate MC immigration. ${ }^{16,27}$ Evidently; most of the neoangiogenesisstimulating factors e.g. the fibroblast growth factor, platelet-derived endothelial cell growth factor, transforming growth factor, vascular endothelial growth factor, etc. have also been demonstrated to be formed by tumor itself or stromal cells.

\section{The dual effect of Mast cell's mediators on neoangiogenesis}

While TNF- $\alpha$, tryptase and cathepsin $G$ are confirmed as the angiogenesis-stimulating factors; particularly TNF- $\alpha$ and cathepsin $\mathrm{G}$ are also thought to mediate $\mathrm{MCC}$ against murine tumor and target cells. ${ }^{30,31,36}$ Inhibition of MCC by a tumor cell product 'squamous cell carcinoma antigen 2' was implicated in the development of advanced squamous cell tumors. ${ }^{37}$ Cathepsin $\mathrm{G}$ is recognized as cytotoxic to some mammalian cells, resembling other serine proteinases in the azurophilic granule, excluding its bactericidal effect against Staphylococcus aureus and Pseudomonas aeruginosa. ${ }^{36}$ Latest findings concerning cathepsin $\mathrm{G}$ involve its cytotoxic capacity against target cells, compatible with previous researches in murine tumor cell lines. ${ }^{30,31}$ Additionally, immediate degradation of MC mediators e.g. cytokines and TNF- $\alpha$ after released was recently shown. Chymase and cathepsin $\mathrm{G}$ were recognized as the possible perpetrators digesting these cytokines. ${ }^{38}$ Therefore, MC's chymase and cathepsin $\mathrm{G}$ in actuality avert the effects of their own mediators signifying that MCs alone are not able to mediate neoangiogenesis in the tumor tissues. Previous researches demonstrated no considerable distinction between MC-deficient and sufficient mice for the angiogenesis. ${ }^{39}$ Nevertheless, besides the capacity of MCs to destroy tumor and 
endothelial cells, new studies also showed no considerable relation between angiogenesis and MCD in human cancers such as in ovarian / renal cancer, regardless of NSCLC. 1,21,22,27,28

\section{Conflicting data for evaluation of Mast cell's role in tumor stroma from the literature}

Although it is tough to elucidate these contradictory results in the literature, they may be attributable to broad distinction in chosen tumor type, stage and evaluation zones of tumor tissues as well as methodologies utilized for demonstration of MCs, vessels, and inflammatory cells. Similarly, any uniform method is not yet available to evaluate the angiogenesis dependably. ${ }^{40}$ If $\mathrm{MC}$ has to do something with neoangiogenesis, it seems to be a less important effect, and I do not think this accessory effect inevitably causes tumor growth. I think additional descriptive in vitro/in vivo researches are essential pertaining to the association of MCs with angiogenesis and tumor cells. Mast cell density, neoplastic angiogenesis and their importance in tumor expansion as well as in prognosis are newly discussed by us with a particular point of view. ${ }^{4-7}$

\section{Conclusion}

I myself consider that the inhibitory or promoting effect of MCs on tumor growth relies on numerous connections among MC type, tumor cell type and the microenvironment in tumor tissues. As anyone can imagine, in this hectic conditions and microenvironments, the MC's authentic role in inflammatory tissue and on neoangiogenesis is very hard to describe. Mast cells might be a new target for the adjuvant treatment of various cancers through the selective inhibition of neoangiogenesis, tissue remodeling and tumor promoting mediators. ${ }^{41,42}$

\section{Acknowledgments}

None.

\section{Conflicts of interest}

None.

\section{References}

1. Ozdemir O. Flow cytometric mast cell mediated cytotoxicity assay:a new three-color flow cytometric approach using monoclonal antibody staining with annexin v/propidium iodide co-labeling to assess human mast cell-mediated cytotoxicity by fluorosphere-adjusted counts. $J$ Immunol Methods. 2011;365(1-2):166-173.

2. Ozdemir O. Evaluation of human mast cell mediated cytotoxicity by DIOC18 target cell labeling in flow cytometry. J Immunol Methods 2007;319(1-2):98-103.

3. Ozdemir O. The role of mast cell density in tumor-associated angiogenesis and survival of squamous cell carcinoma of the lung. $J$ Cancer Res Ther. 2015;11(4):1041.

4. Ozdemir O. Might mast cells have a role in neoplastic angiogenesis of canine melanomas? Vet Dermatol. 2006;17(4):284-289.

5. Ozdemir O. Mast cells and the tumor associated neoangiogenesis. Med Sci Monit. 2006;12(6):LE9-11.

6. Ozdemir O. Mast cell density, angiogenesis, and their significance in tumor development. Gynecol Oncol. 2006;100(3):628-629.

7. Ozdemir O. Mast cell density, neoplastic angiogenesis and their prognostic importance. Dig Liver Dis. 2006;38(5):356.

8. Ozdemir O, Dağoğlu R, Goksu Erol A. Antitumor actions of human mast cells. Pol J Pathol. 2012;63(4):292.
9. Ozdemir O. Immunosurveillance function of human mast cell? World $J$ Gastroenterol. 2005;11(44):7054-7056.

10. Krystel Whittemore M, Dileepan KN, Wood JG. Mast Cell:A Multi Functional Master Cell. Front Immunol. 2016;6:620.

11. Moon TC, Befus AD, Kulka M. Mast cell mediators:their differential release and the secretory pathways involved. Front Immunol. 2014;5:569.

12. Frossi B, Mion F, Tripodo C, et al. Rheostatic functions of mast cells in the control of innate and adaptive immune responses. Trends Immunol. 2017;pii:S1471-4906(17)30060-1.

13. Hiromatsu Y, Toda S. Mast cells and angiogenesis. Microsc Res Tech 2003;60(1):64-69.

14. Yamazaki A, Nakamura T, Omori K, et al. The role of mast cell in inflammation. Nihon Yakurigaku Zasshi. 2017;149(5):204-207.

15. Aldinucci D, Gloghini A, Pinto A, et al. The classical Hodgkin's lymphoma microenvironment and its role in promoting tumour growth and immune escape. J Pathol. 2010;221(3):248-263.

16. Gruber BL, Marchese MJ, Kew R. Angiogenic factors stimulate mas cell migration. Blood. 1995;86(7):2488-2493.

17. Väyrynen JP, Tuomisto A, Klintrup K, et al. Detailed analysis of inflammatory cell infiltration in colorectal cancer. $\mathrm{Br} J$ Cancer 2013;109(7):1839-1847.

18. Caruso RA, Fedele F, Rigoli L. Mast cell interaction with tumor cells in small early gastric cancer:ultrastructural observations. Ultrastructural Pathology. 1997;21(2):173-181.

19. Ohri CM, Shikotra A, Green RH, et al. Chemokine receptor expression in tumour islets and stroma in non-small cell lung cancer. BMC Cancer 2010;10:172.

20. Chan JK, Magistris A, Loizzi V, et al. Mast cell density, angiogenesis, blood clotting, and prognosis in women with advanced ovarian cancer Gynecol Oncol. 2005;99(1):20-25.

21. Raica M, Cimpean AM, Ceausu R, et al. Interplay between mast cells and lymphatic vessels in different molecular types of breast cancer Anticancer Res. 2013;33(3):957-963.

22. Grizzi F, Franceschini B, Chiriva Internati M, et al. Mast cells and human hepatocellular carcinoma. World J Gastroenterol. 2003;9(7):1469-1473.

23. Ponvert C, Galoppin L, Canu P. Modifications in tissue histamine levels in mast cell-deficient mice $(\mathrm{W} / \mathrm{Wv})$ and in their littermates $(\mathrm{Wv} /+, \mathrm{W} /+$ and $+/+)$ grafted with a methylcholanthrene-induced fibrosarcoma:correlation with tumour rejection. Cancer Lett. 1994;85(1):125-131.

24. Burtin C, Ponvert C, Fray A, et al. Inverse correlation between tumor incidence and tissue histamine levels in $\mathrm{W} / \mathrm{WV}, \mathrm{WV} /+$, and $+/+$ mice. $J$ Natl Cancer Inst. 1985;74(3):671-674.

25. Schittek A, Issa HA, Stafford JH, et al. Growth of pulmonary metastases of B16 melanoma in mast cell-free mice. J Surg Res. 1985;38(1):24-28.

26. Van Loveren H, Den Otter W, Meade R, et al. A role for mast cells and the vasoactive amine serotonin in T cell-dependent immunity to tumors. J Immunol. 1985;134(2):1292-1299.

27. Carlini MJ, Dalurzo MC, Lastiri JM, et al. Mast cell phenotypes and microvessels in non small cell lung cancer and its prognostic significance. Hum Pathol. 2010;41(5):697-705

28. Dundar E, Oner U, Peker BC, et al. The significance and relationship between mast cells and tumour angiogenesis in non-small cell lung carcinoma. J Int Med Res. 2008;36(1):88-95.

29. Prior C, Sesenna R. Mast cells and their relation to the tumors of the bladder. Riv Anat Patol Oncol. 1953;7:809-838. 
30. Clarke GR, Shirzadeh H, Pang G, et al. TNF alpha is not the sole mediator of WEHI-164 tumour cell killing in natural cytotoxicity. Cytokine. 1997;9(4):254-262.

31. Shirzadeh H, Clarke GR, McNeil HP, et al. An IL-3-induced splenic $\mathrm{NC}-1.1+$ mast cell line mediates natural cytotoxicity independent of TNF-alpha. Cell Immunol. 1996;174(2):147-154.

32. Leskinen MJ, Lindstedt KA, Wang Y, et al. Mast cell chymase induces smooth muscle cell apoptosis by a mechanism involving fibronectin degradation and disruption of focal adhesions. Arterioscler Thromb Vasc Biol. 2003;23(2):238-243.

33. Samoszuk M, Kanakubo E, Chan JK. Degranulating mast cells in fibrotic regions of human tumors and evidence that mast cell heparin interferes with the growth of tumor cells through a mechanism involving fibroblasts. BMC Cancer. 2005;5:121.

34. Theoharides TC, Conti P. Mast cells:the Jekyll and Hyde of tumor growth. Trends Immunol. 2004;25:235-241.

35. Lätti S, Leskinen M, Shiota N, et al. Mast cell mediated apoptosis of endothelial cells in vitro:a paracrine mechanism involving TNF alpha mediated down-regulation of bcl-2 expression. J Cell Physiol. 2003;195(1):130-138.

36. Heutinck KM, Ten Berge IJ, Hack CE, et al. Serine proteases of the human immune system in health and disease. Mol Immunol. 2010;47(11-12):1943-1955.
37. Schick C, Kamachi Y, Bartuski AJ, et al. Squamous cell carcinoma antigen 2 is a novel serpin that inhibits the chymotrypsin like proteinases cathepsin G and mast cell chymase. J Biol Chem. 1997;272(3):18491855 .

38. Zhao W, Oskeritzian CA, Pozez AL, et al. Cytokine production by skinderived mast cells:endogenous proteases are responsible for degradation of cytokines. J Immunol. 2005;175(4):2635-2642.

39. Ghosh AK, Hirasawa N, Ohtsu H, et al. Defective angiogenesis in the inflammatory granulation tissue in histidine decarboxylase deficient mice but not in mast cell deficient mice. J Exp Med. 2002;195:973-982.

40. Ciocâlteu A, Săftoiu A, Cârţână T, et al. Evaluation of new morphometric parameters of neoangiogenesis in human colorectal cancer using confocal laser endomicroscopy (CLE) and targeted panendothelial markers. PLoS One. 2004;9(3):e91084

41. Cimpean AM, Tamma R, Ruggieri S, et al. Mast cells in breast cancer angiogenesis. Crit Rev Oncol Hematol. 2017;115:23-26.

42. Varricchi G, Galdiero MR, Loffredo S, et al. Are Mast cells masters in cancer? Front Immunol. 2017;8:424. 